\title{
Uncertainty Propagation and the Fano Based Information Theoretic Method
}

\author{
John A. Malas Senior Member IEEE, John A. Cortese, and Patricia Ryan, Senior Member, IEEE
}

\begin{abstract}
The Fano equality is joined with the data-processing inequality to develop a theory model for component level trade studies within radar signature exploitation systems. Entropy is used to represent propagating uncertainty within an information channel. Measures are developed to identify information flow bottlenecks within an information loss budget. The propagating effects of various sources of uncertainty on system performance are characterized.
\end{abstract}

Index Terms-Information Theory, Uncertainty Propagation, Radar.

\section{INTRODUCTION}

【 nnovations in radar sensing are spurring new research in the area of target signature measurement and exploitation. The ability to characterize information extraction while under the effects of system uncertainties is critical to risk based design methods. The use of existing systems theory prototypes such as the radar range equation is inadequate in fully characterizing the flow of information through the sensing system. Information theory prototypes enable the study of the propagating effects of various sources of uncertainty on system performance at the point of noise infiltration.

\section{A. Historical Contributions}

Woodward and Davies [1] and Woodward [2] were the first to apply the information theoretic approach to the analysis of radar soon after the appearance of Shannon's original work [3] on information theory. More recently Bell [4] has suggested the use of an information theoretic approach to the design of radar waveforms. Dr. Bell formulated and obtained a solution to the problem of designing a waveform that maximized the MI between the target impulse response and the received signal. Recently, Leshem et al. [5] extended Bell's work to the case of multiple extended targets. Others have applied the principles of information theory to the area of radar target identification [6-16]. The area of uncertainty modeling and sensitivity analysis is wide ranging, drawing upon established fields such as the design of experiments [17] and classical engineering methods of statistics that lead to

This work was supported in part by the Air Force Office of Scientific Research under Grant 10RY08COR

J. A. Malas is with the Air Force Research Laboratory, WPAFB, OH 45433 USA, 937-528-8215; fax: 303-555-5555; john.malas@wpafb.af.mil.

P. Ryan is with is with the Air Force Research Laboratory, WPAFB, OH 45433 USA; patricia.ryan@wpafb.af.mil .

J. A. Cortese is with Massachusetts Institute of Technology, Lincoln Laboratory, 244 Wood Street, Lexington, MA, 02420; icort@11.mit.edu . uncertainty measures [18], including the traditional methods of Taylor Series expansion and differential calculus [19]. Recently, advances in large scale computer simulation have opened the door to modeling complex physical processes in lieu of expensive physical experiments [20]. The methods by Saltelli et al. [21] present new Monte-Carlo Methods for the study of sensitivity.

\section{APPROACH}

\section{A. Systems Theory Model and Uncertainty}

Using Fano's inequality, the max flow [15] criteria bounds the optimal Bayes error. Entropy and mutual information (MI) are analytically connected to the probability of error $\left(\mathrm{P}_{\mathrm{e}}\right)$ and more generally the Neyman Pearson criteria allowing for the rate of noise infiltration to be related to the rate of entropy growth and ultimately to the rate of degradation of system performance. The information loss associated with uncertainty sources can be characterized in terms of a confidence or reliability interval about the predicted system performance at each component of the system. The data processing inequality affords a method to determine information loss points and maximize information flow via component trades within a system information loss budget. An abbreviated and preliminary treatment of this concept is presented in [22]. The method is applied to a multidisciplinary problem set to study the relative significance of various "unknown" operating conditions using Monte-Carlo simulation experiments.

\section{B. Sensing Uncertainty Example}

A related problem of interest is the measurement of airborne moving objects using high range resolution (HRR) waveforms. Two classes of system uncertainty are given in Table I as Sensing uncertainty and uncertainty resulting from Decision Rule Training Limitations.

The object under measurement by the sensing radar system can be viewed as a collection of scattered field sources filling an electrically large volume in space. The measurement of this object is subject to uncertainty identified in source 1.a at a fixed position in time. Target fixed body motion within the measurement interval induces scintillation within the scattering sources resulting in an additional increase in entropy. Imperfect knowledge of target position, velocity, and aspect angle also alters the statistical characterization of the random signature process (source 1.c). In addition, the random signature process interacts with an external environment (source 1.b) to further impact the statistical nature of the measured signature process. 
TABle I. RAdar System UnCERTAinty SourceS

\begin{tabular}{|l|c|c|c|}
\hline \multicolumn{1}{|c|}{$\begin{array}{c}\text { Uncertainty Core } \\
\text { Area }\end{array}$} & \multicolumn{3}{|c|}{ Parameter Uncertainty Subcategory } \\
\cline { 1 - 1 } 1. Sensing & \multirow{2}{*}{$\begin{array}{c}\text { Non-linear } \\
\text { Effects } \\
\text { a. Signature } \\
\text { Measurement }\end{array}$} & $\begin{array}{c}\text { I\&Q } \\
\text { Quantization/ } \\
\text { Clipping }\end{array}$ & $\begin{array}{c}\text { Amplitude \& } \\
\text { Phase } \\
\text { Calibration }\end{array}$ \\
\cline { 1 - 2 } b.Environmental & $\begin{array}{c}\text { Clutter/Therm } \\
\text { al Noise }\end{array}$ & $\begin{array}{c}\text { RF } \\
\text { Interference }\end{array}$ & Jamming \\
\cline { 1 - 2 } $\begin{array}{l}\text { c. Object } \\
\text { Tracking \& } \\
\text { Motion }\end{array}$ & $\begin{array}{c}\text { Object Range, } \\
\text { Velocity, \& } \\
\text { Aspect } \\
\text { Estimates }\end{array}$ & $\begin{array}{c}\text { Object } \\
\text { Articulation }\end{array}$ & $\begin{array}{c}\text { Intra- } \\
\text { measurement } \\
\text { Motion }\end{array}$ \\
\cline { 1 - 2 } $\begin{array}{l}\text { 2. Decision Rule } \\
\text { Training } \\
\text { Limitations }\end{array}$ & $\begin{array}{c}\text { Process Under } \\
\text { Sampling }\end{array}$ & $\begin{array}{c}\text { Target } \\
\text { Configuration } \\
\text { Variation }\end{array}$ & $\begin{array}{c}\text { Target } \\
\text { Modeling } \\
\text { Parameters }\end{array}$ \\
\hline
\end{tabular}

Only a subset of the phenomenon (parameters) underlying source 1 can be modeled and/or characterized within the decision rule training process resulting in a decision rule design that is less than optimal with respect to system performance.

\section{Information Theoretic Sensor Channel}

The radar information sensing system can be viewed within a discrete memoryless broadcast channel depicting the information flow through the sensing and processing components of a radar system as shown in Fig. 1 [22], [23].

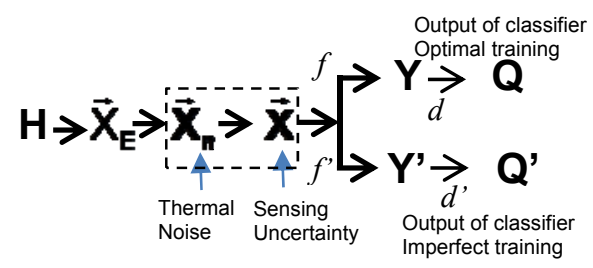

Fig. 1. Information Theoretic Sensor Broadcast Channel

The discrete random variable $\mathbf{H}$ represents which of the $\mathrm{N}_{c}$ possible hypotheses has occurred. The discrete random variable $\mathbf{Q}$ is of the same alphabet as $\mathbf{H}$. Successful flow of information results in agreement between $\mathbf{H}$ and $\mathbf{Q}$. Conditioned on the generating hypothesis $\mathbf{H}$ (instance of $\mathbf{H}$ ), there is typically an encoded source $\vec{X}_{E}$ which is realized as the image projection of the object under measurement. In the case of an HRR radar measurement, this is the Fourier transform of the band-limited frequency response associated with the scattered field of the observed object in thermal noise. After mixing, filtering, and signal processing, these returns become the measured random signature vector $\overrightarrow{\mathbf{X}}_{n}$. The sensing of $\overrightarrow{\mathbf{X}}_{n}$ is subjected to the uncertainties listed in source 1 of Table I leading to the random signature process $\overrightarrow{\mathbf{X}}$.

The multivariate sample feature $\vec{Y}$ is extracted from the $i^{\text {th }}$ instance test sample $\vec{X}$ to support the desired function of the exploitation system. Given the random nature of $\overrightarrow{\mathbf{X}}$, the extracted signature feature $\overrightarrow{\mathbf{Y}}$ is also random. The training feature process $\overrightarrow{\mathbf{Y}}^{\prime}$ is developed from the set of typical signatures within the surrogate decision rule training process
$\overrightarrow{\mathbf{X}}$. The decision algorithm applies $\vec{Y}$ to the decision rule $d$ yielding the decision $\mathbf{Q}$ (instance of $\mathbf{Q}$ ) declaring which of the hypotheses has occurred. The evaluation of an ensemble $N_{M}$ of test samples $\vec{X}\left\{i=1 \rightarrow N_{M}\right\}$ produces the sample ensemble of the $\mathrm{N}_{M}$ matching tests $(\mathrm{H}, \mathrm{Q})$ to statistically characterize the decision performance.

\section{THEORY}

\section{A. Fano Based Information Theoretic Method (FBIT)}

Fano's inequality [23] provides a mathematical means to relate the MI between $\mathbf{H}$ and $\mathbf{Q}, \mathrm{I}(\mathbf{H} ; \mathbf{Q})$, to a lower bound on $P_{e}$ and can be written as an equality as in (1) [22].

$$
\mathrm{H}\left(\mathrm{P}_{\mathrm{e}}\right)=\delta-\mathbf{P}_{\mathrm{e}} \cdot \log \left(\mathrm{N}_{\mathrm{c}}-1\right)+\mathrm{H}(\mathbf{H})-\mathrm{I}(\mathbf{H} ; \mathbf{Q})
$$

In (1), $P_{e}$ is a real random variable between 0 and .5 representing the probability of error of the decision algorithm. $\mathrm{N}_{\mathrm{c}}$ is the discrete size of the alphabet of $\mathbf{H}$ and $\mathbf{Q}$. $\mathrm{H}(\mathbf{H})$ is the Shannon entropy of the discrete random variable $\mathbf{H}$ corresponding to the different target classes. The asymmetry factor $\delta$ is a bias offset [24] fixed by architectural considerations that can be computed directly from the output of the decision algorithm. Let $\delta=\mathrm{I}(\mathbf{Q} ; \mathbf{V})$ for $\mathrm{N}_{\mathrm{c}}=2$; where $\mathbf{V}$ is the binary discrete random variable where $\mathbf{V}=1$ when $\mathbf{H}=\mathbf{Q}$; otherwise $\mathbf{V}=0$. Equation (1) can be written in terms of the inverse entropy function $\mathrm{F}$ as shown in (2) for equiprobable hypothesis case $N_{c}=2 . F$ is a deterministic strictly monotonically increasing function

$$
\mathrm{P}_{\mathrm{e}}=\mathrm{F}(\mathrm{H}(\mathbf{H})-\mathrm{I}(\mathbf{H} ; \mathbf{Q})+\mathrm{I}(\mathbf{Q} ; \mathbf{V}))
$$

The Data Processing Inequality [23] states that information can only be lost in the channel as shown in (3).

$$
\mathrm{I}(\mathbf{H} ; \overrightarrow{\mathbf{X}}) \geq \mathrm{I}(\mathbf{H} ; \overrightarrow{\mathbf{Y}}) \geq \mathrm{I}(\mathbf{H} ; \mathbf{Q})
$$

Using the relationship in (3), the loss associated with each link within the channel can be characterized as in (4).

$$
\mathrm{H}(\mathbf{H})-\mathrm{I}(\boldsymbol{H} ; \overrightarrow{\mathbf{X}}) \leq \mathrm{H}(\mathbf{H})-\mathrm{I}(\boldsymbol{H} ; \overrightarrow{\mathbf{Y}}) \leq \mathrm{H}(\mathbf{H})-\mathrm{I}(\mathbf{H} ; \boldsymbol{Q}) .
$$

The cumulative information loss at each link in the channel can then be written as below applying (3).

$$
\begin{array}{r}
\mathrm{IL}_{\overrightarrow{\mathbf{X}}}=\mathrm{H}(\mathbf{H})-\mathrm{I}(\mathbf{H} ; \overrightarrow{\mathbf{X}}) ; \quad \overrightarrow{\mathbf{X}} \in|\chi| \\
\mathrm{IL}_{\overrightarrow{\mathbf{Y}}}=\mathrm{H}(\mathbf{H})-\mathrm{I}(\mathbf{H} ; \overrightarrow{\mathbf{Y}}) ; \overrightarrow{\mathbf{Y}} \in|Y| \\
\mathrm{IL}_{\mathbf{Q}}=\mathrm{H}(\mathbf{H})-\mathrm{I}(\mathbf{H} ; \mathbf{Q}) ; \quad \mathbf{Q} \in|Q|
\end{array}
$$

Since $F$ is a known function, the deterministic relation $P_{e}=$ $\mathrm{F}(\mathrm{H}(\mathbf{H})-\mathrm{I}(\mathbf{H} ; \mathbf{Q})+\mathrm{I}(\mathbf{Q} ; \mathbf{V}))$, for fixed $\mathrm{H}(\mathbf{H})$ and $\delta$, determines the MI $(\mathrm{I}(\mathbf{H} ; \mathbf{Q}))$ needed to achieve a specified $\mathrm{P}_{\mathrm{e}}$. Specifying a desired $\mathrm{P}_{\mathrm{e}}$ determines the amount of allowed $\mathrm{IL}_{Q}$ via component (link) design. The respective information loss due to each link within the Markov chain $\mathbf{H} \rightarrow \mathbf{X} \rightarrow \mathbf{Y} \rightarrow \mathbf{Q}$ can then be quantified using equations $(6 a-6 c)$.

$$
\begin{array}{r}
\text { Loss due to Sensing } \equiv \mathrm{IL}_{\mathrm{S} \Delta}=\mathrm{H}(\mathbf{H})-\mathrm{I}(\mathbf{H} ; \overrightarrow{\mathbf{X}}) \\
\text { Loss due to Feature Extraction } \equiv \mathrm{IL}_{\mathrm{F} \Delta}=\mathrm{I}(\mathbf{H} ; \overrightarrow{\mathbf{X}})-\mathrm{I}(\mathbf{H} ; \overrightarrow{\mathbf{Y}}) \\
\text { Loss due to Decision Rule } \equiv \mathrm{IL}_{\mathrm{D} \Delta}=\mathrm{I}(\mathbf{H} ; \overrightarrow{\mathbf{Y}})-\mathrm{I}(\mathbf{H} ; \mathbf{Q})
\end{array}
$$


Under conditions such as those listed in uncertainty source 2 in Table I, the surrogate representation $\overrightarrow{\mathbf{X}}^{\prime}$ used in the training of the decision rule results in a non-optimal decision rule $d$ '. This is represented by the altered entropic quantity $\mathrm{H}\left(\mathbf{Q}^{\prime}\right)$ and more importantly I(H;Q'). The corresponding form of (4) can then be written as

$$
\mathrm{H}\left(\mathbf{P}_{\mathbf{e}}{ }^{\prime}\right)=\mathrm{H}(\mathbf{H})-\mathrm{I}\left(\mathbf{H}_{\mathbf{Q}^{\prime}}\right)+\mathrm{I}\left(\mathbf{Q}^{\prime} ; \mathbf{V}\right)=\mathrm{IL}_{\mathbf{Q}^{\prime}}+\mathrm{I}\left(\mathbf{Q}^{\prime} ; \mathbf{V}\right) \text {. }
$$

Therefore since $\mathrm{H}\left(\mathbf{P}_{\mathbf{e}}^{\prime}\right) \geq \mathrm{H}\left(\mathbf{P}_{\mathbf{e}}\right), \mathrm{I}\left(\mathbf{H}_{\mathbf{Q}} \mathbf{Q}^{\prime}\right)-\mathrm{I}\left(\mathbf{Q}^{\prime} ; \mathbf{V}\right) \leq \mathrm{I}(\mathbf{H} ; \mathbf{Q})$ $\mathrm{I}(\mathbf{Q} ; \mathbf{V})$. Information loss due to imperfect training, $\mathrm{IL}_{\mathrm{T} \Delta}$, is then mathematically quantified in terms of the increase in entropy $\Delta \mathrm{H}\left(\mathbf{P}_{\mathbf{e}}\right) ; \quad I L \quad{ }_{\mathrm{T}}=\Delta \mathrm{H}\left(\mathbf{P}_{\mathbf{e}}\right)=\mathrm{H}\left(\mathbf{P}_{\mathbf{e}}^{\prime}\right)-\mathrm{H}\left(\mathbf{P}_{\mathbf{e}}\right)$

$$
=-\mathrm{I}\left(\mathbf{H}_{\mathbf{Q}} \mathbf{Q}^{\prime}\right)+\mathrm{I}\left(\mathbf{Q}^{\prime} ; \mathbf{V}\right)+\mathrm{I}(\mathbf{H} ; \mathbf{Q})-\mathrm{I}(\mathbf{Q} ; \mathbf{V}) \text {. }
$$

If it can be shown that $\mathrm{I}(\mathbf{Q} ; \mathbf{V}) \cong \mathrm{I}\left(\mathbf{Q}^{\prime} ; \mathbf{V}\right)$ and that

$\mathrm{I}(\mathbf{Q} ; \mathbf{V})<<\mathrm{H}(\mathbf{H})-\mathrm{I}(\mathbf{H} ; \mathbf{Q})$ and $\mathrm{I}\left(\mathbf{Q}^{\prime} ; \mathbf{V}\right)<<\mathrm{H}(\mathbf{H})-\mathrm{I}\left(\mathbf{H}_{\mathbf{2}} \mathbf{Q}^{\prime}\right)$ then;

$$
\text { Imperfect Training Loss } \equiv \mathrm{IL}_{\mathrm{T} \Delta} \cong \mathrm{I}(\mathbf{H} ; \mathbf{Q})-\mathrm{I}\left(\mathbf{H} ; \mathbf{Q}^{\prime}\right) .
$$

The total loss in the channel is equal to the sum of all link loss components plus $\mathrm{IL}_{\mathrm{T} \Delta}$.

$$
\mathrm{IL}_{\text {Total }}=\mathrm{IL}_{\mathrm{S} \Delta}+\mathrm{IL}_{\mathrm{F} \Delta}+\mathrm{IL}_{\mathrm{D} \Delta}+\mathrm{IL}_{\mathrm{T} \Delta}
$$

Thus, the probability of error can be estimated at various points in the channel using the approximations;

$$
\begin{aligned}
& \mathrm{P}_{\mathrm{e}}^{\mathrm{X}} \approx \mathrm{F}(\mathrm{H}(\mathbf{H})-\mathrm{I}(\mathbf{H} ; \overrightarrow{\mathbf{X}})) \\
& \mathrm{P}_{\mathrm{e}}{ }^{\mathrm{Y}} \approx \mathrm{F}(\mathrm{H}(\mathbf{H})-\mathrm{I}(\mathbf{H} ; \overrightarrow{\mathbf{Y}})) \\
& \mathrm{P}_{\mathrm{e}}{ }^{\mathrm{Q}} \approx \mathrm{F}(\mathrm{H}(\mathbf{H})-\mathrm{I}(\mathbf{H} ; \mathbf{Q})) \\
& \mathrm{P}_{\mathrm{e}}{ }^{\mathrm{Q}^{\prime}} \approx \mathrm{F}\left(\mathrm{H}(\mathbf{H})-\mathrm{I}\left(\mathbf{H} ; \mathbf{Q}^{\prime}\right)\right) .
\end{aligned}
$$

\section{B. Propagating Effects of Sensor Uncertainty}

\section{a) Propagating Link Loss}

Uncertainty propagation is the study of how uncertainty in the output of a model (numerical or otherwise) can be apportioned to different sources of uncertainty in the model inputs [21]. As shown in Fig. 1, the propagating effects of uncertainty will depend on the point of entry in the channel.

The vector $\vec{v}_{c}$ represents the sensor control parameters of interest and provides the deterministic mapping function $\mathrm{P}_{\mathrm{e}}^{X}\left(\vec{v}_{c}\right)$ in (10.a). The distribution parameters associated with environmental random input parameters of environmental $\left(\vec{V}_{E}\right)$ and target position $\left(\vec{V}_{t}\right)$ uncertainty are estimated from the data and result in the mapping to the random signature process function $\overrightarrow{\mathbf{X}}\left(\vec{v}_{c}, \vec{V}_{E}, \vec{V}_{t}\right)$. The sensing uncertainty is then subsequently propagated into the random feature process function $\overrightarrow{\mathbf{Y}}\left(\vec{v}_{c}, \vec{V}_{E}, \vec{V}_{t}\right)$ and ultimately to the decision process function $\mathbf{Q}\left(\vec{v}_{c}, \vec{V}_{E}, \vec{V}_{t}\right)$. For brevity these random process functions are written as, $\overrightarrow{\mathbf{X}}, \overrightarrow{\mathbf{Y}}$ and $\mathbf{Q}$.
The cumulative link information loss as quantified within (5a), (5b), and (5c) then become random variables $\mathbf{I L}_{\overrightarrow{\mathbf{x}}}, \mathbf{I L}_{\overrightarrow{\mathrm{Y}}}$, $\mathbf{I L}_{\mathbf{Q}}$, and $\mathbf{I L}_{\mathbf{Q}}$. Similarly, the link information loss $\mathbf{I L}_{\mathbf{s} \Delta,} \mathbf{I L}_{\mathbf{F} \Delta \text {, }}$ $\mathbf{I L}_{\mathbf{D} \Delta}$, and $\mathbf{I L}_{\mathbf{T} \Delta}$ in (6.a), (6.b), (6.c), and (8) also become random variables. Using Monte Carlo simulation, the individual sources of uncertainty within $\vec{V}_{E}$ and $\vec{V}_{t}$ are treated as independent and thus cumulative link loss variance can be decomposed into the sum of the variance components of each uncertainty source as in the example below at link $\mathrm{X}$ assuming $\mathrm{n}_{\mathrm{e}}$ factors within $\vec{V}_{E}$ and $\mathrm{n}_{\mathrm{t}}$ factors within $\vec{V}_{t}$.

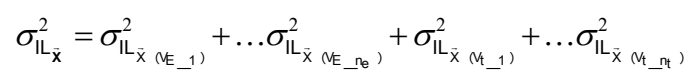

The mean cumulative loss given (12) follows from the linearity of the expectation operation and the additive relationship between the formerly deterministic quantities in (9).

$$
\mu_{\mathrm{IL}_{\text {Total }}}=\mu_{\mathrm{IL}_{\mathrm{s}_{\Delta}}}+\mu_{\mathrm{IL}_{\mathrm{F}_{\Delta}}}+\mu_{\mathrm{IL}_{\mathrm{D}_{\Delta}}}+\mu_{\mathrm{IL}_{\mathrm{T}_{\Delta}}}
$$

\section{b) Propagating Link Loss to Link Performance}

The Maximum Likelihood Estimate of $\mathrm{P}_{\mathrm{e}}$ is inferred at each realization of the statistical space $\left(\vec{v}_{c}, \vec{V}_{E}, \vec{V}_{t}\right)$ providing the random mapping to the link performance functions.

$$
\begin{aligned}
& \mathbf{P}_{\mathrm{e}}^{\overrightarrow{\mathrm{X}}} \equiv \mathrm{P}_{\mathrm{e}}^{\mathrm{X}}\left(\vec{v}_{c}, \vec{V}_{E}, \vec{V}_{t}\right) \approx \mathrm{F}\left(\mathbf{I} \mathbf{L}_{\overrightarrow{\mathbf{x}}}\right) \\
& \mathbf{P}_{\mathbf{e}}^{\overrightarrow{\mathrm{Y}}} \equiv \mathrm{P}_{\mathrm{e}}^{\mathrm{Y}}\left(\vec{v}_{c}, \vec{V}_{E}, \vec{V}_{t}\right) \approx \mathrm{F}(\mathbf{I L} \overrightarrow{\mathrm{Y}})
\end{aligned}
$$

Under training where $\vec{v}_{c}{ }^{\prime}=\vec{v}_{c}, \vec{V}_{E}{ }^{\prime}=\vec{V}_{E}, \vec{V}_{t}{ }^{\prime}=\vec{V}_{t}$, then the mapping to the decision rule $d_{o p t}$ will be optimal as in (15).

$$
\mathbf{P}_{\mathbf{e}}^{\mathbf{Q}} \equiv \mathbf{P}_{\mathbf{e}}{ }^{\mathbf{Q}}\left(\vec{v}_{c}, \vec{V}_{E}, \vec{V}_{t}\right)=\mathrm{F}\left(\mathbf{I L}_{\mathbf{Q}}+\mathrm{I}(\mathbf{Q} ; \mathbf{V})\right) \approx \mathrm{F}\left(\mathbf{I L}_{\mathbf{Q}}\right)
$$

For non-optimal conditions $\vec{V}_{E}{ }^{\prime} \neq \vec{V}_{E}$ and $\vec{V}_{t}^{\prime} \neq \vec{V}_{t}$, the performance mapping function at $Q^{\prime}$ is represented in (16).

$$
\begin{aligned}
\mathbf{P}_{\mathbf{e}}^{\mathbf{Q}^{\prime}} \equiv \mathbf{P}_{\mathbf{e}}^{\mathbf{Q}^{\prime}}\left(\vec{v}_{c}, \vec{V}_{E^{\prime}}, \vec{V}_{t}^{\prime}\right) & =\mathrm{F}\left(\mathrm{H}(\mathbf{H})-\mathrm{I}\left(\mathbf{H} ; \mathbf{Q}^{\prime}\right)+\mathrm{I}\left(\mathbf{Q}^{\prime} ; \mathbf{V}^{\prime}\right)\right) \\
& \approx \mathrm{F}\left(\mathbf{I L}_{\mathbf{Q}^{\prime}}\right)=\mathrm{F}\left(\mathrm{H}(\mathbf{H})-\mathrm{I}\left(\mathbf{H} ; \mathbf{Q}^{\prime}\right)\right)
\end{aligned}
$$

The expected link performance under control parameters $\vec{v}_{c}$ and in the presence of sensing uncertainty $\left(\vec{V}_{E}, \vec{V}_{t}\right)$ is defined as the expectation of the random link performance

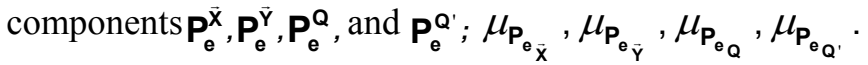

Reliability in predicted link performance is defined as the standard deviation $\left(\sigma_{\mathrm{P}_{\bar{e}}^{\bar{x}}}, \sigma_{\mathrm{P}_{\mathrm{e}}^{\bar{y}}}, \sigma_{\mathrm{P}_{\mathrm{e}}^{Q}}\right.$, and $\left.\sigma_{\mathrm{P}_{\mathrm{e}}^{Q^{\prime}}}\right)$ of the respective random cumulative link performance associated with $\mathbf{P}_{\mathbf{e}}^{\overrightarrow{\mathbf{x}}}, \mathbf{P}_{\mathbf{e}}^{\overline{\mathbf{Y}}}, \mathbf{P}_{\mathbf{e}}^{\mathbf{Q}}$, and $\mathbf{P}_{\mathbf{e}}^{\mathbf{Q}^{\prime}}$. The variability in link performance is defined as the square of the reliability. 


\section{Uncertainty in Performance}

It is desired to relate the independent sources of uncertainty underlying the variance in cumulative link information loss to the corresponding set of variances that combine to equal the variability in link performance in order to directly relate information loss to performance reliability. While this relationship is transcendental and nonlinear, when the uncertainty is small and tight about the mean, it is possible to approximate the inverse entropy function $(\mathrm{F})$ by a linear relationship [25] about the mean of $\mathbf{I L}_{\overrightarrow{\mathbf{x}}} ; \mathrm{F}\left(\mathbf{I L}_{\overrightarrow{\mathbf{x}}}\right)=\mathbf{a}+\mathbf{b} \cdot\left(\mathbf{I} \mathbf{L}_{\overrightarrow{\mathbf{x}}}\right)$. Using link $X$ as an example, the mean and variance of the approximation are then $E\left[\mathrm{~F}\left(\mathbf{I} \mathbf{L}_{\overrightarrow{\mathbf{x}}}\right)\right]=\mathrm{a}+\mathrm{b} \cdot\left\{\mu_{\mathbf{I L}_{\overrightarrow{\mathbf{x}}}}\right\}$ and

$\operatorname{Var}[\mathrm{F}(\mathbf{I L} \overrightarrow{\mathbf{x}})]=\mathrm{b}^{2} \cdot\left\{\sigma_{\mathbf{I L}_{\overline{\mathbf{x}}}}^{2}\right\}$. The first order Taylor expansion of

F around the mean $\mu_{\mathrm{IL}_{\overline{\mathbf{x}}}}$ of $\mathbf{I L}_{\overrightarrow{\mathbf{x}}}$ is equal to

$$
\mathrm{F}\left(\mathbf{I L}_{\overrightarrow{\mathbf{x}}}\right) \approx \mathrm{F}\left(\mu_{\mathrm{IL}_{\overrightarrow{\mathrm{x}}}}\right)+\mathrm{F}^{\prime}\left(\mu_{\mathrm{IL}_{\overrightarrow{\mathrm{x}}}}\right) \cdot\left\{\mathbf{I L}_{\overrightarrow{\mathbf{x}}}-\mu_{\mathrm{IL}_{\overrightarrow{\mathrm{x}}}}\right\} .
$$

The approximations for $E\left[\mathrm{~F}\left(\mathbf{I} \mathbf{L}_{\overrightarrow{\mathbf{x}}}\right)\right]$ and $\operatorname{Var}[\mathrm{F}(\mathbf{I L} \overrightarrow{\mathbf{x}})]$ are [25]

$$
\begin{aligned}
& E\left[\mathrm{~F}\left(\mathbf{I L}_{\overrightarrow{\mathbf{x}}}\right)\right]=E\left[\mathbf{P}_{e}^{\overrightarrow{\mathbf{x}}}\right] \approx \mathrm{F}\left(\mu_{\mathbf{I L}_{\overrightarrow{\mathbf{x}}}}\right)=\mathrm{H}^{-1}\left(\mu_{\mathbf{I L}_{\vec{x}}}\right) . \\
& \operatorname{Var}\left[\mathrm{F}\left(\mathbf{I L}_{\overrightarrow{\mathbf{x}}}\right)\right]=\sigma_{\mathrm{P}_{e}^{\vec{x}}}^{2} \approx\left[\mathrm{F}^{\prime}\left(\mu_{\mathbf{I L}_{\overrightarrow{\mathbf{x}}}}\right)\right]^{2} \cdot\left\{\sigma_{\mathbf{L L}_{\overline{\mathbf{x}}}}^{2}\right\}
\end{aligned}
$$

and $\mathrm{F}^{\prime}\left(\mu_{\mathrm{IL}_{\overline{\mathrm{x}}}}\right)$ can be shown to equal

$$
F^{\prime}\left(\mu_{L_{x}}\right)=\log \left[\frac{1}{\left[\frac{\mathrm{H}^{-1}\left(\mu_{L_{L_{x}}}\right)}{1-\mathrm{H}^{-1}\left(\mu_{L_{x}}\right)}\right]}\right]^{.}
$$

The cumulative link loss variance components are applied.

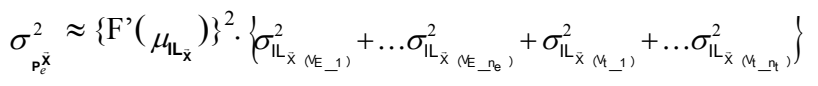

The variability in link performance is then decomposed into the individual sources of uncertainty being propagated at $\overrightarrow{\mathbf{X}}$.

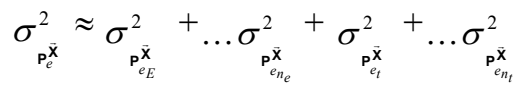

Similar methods can be applied to the independent contributions to the sensing uncertainty of $\vec{V}_{E}$ and $\vec{V}_{t}$

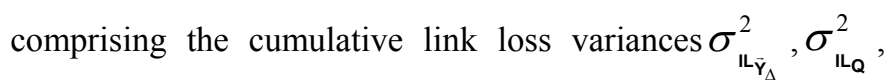
and $\sigma_{\mathrm{IL}_{\mathbf{Q}^{\prime}}}^{2}$ at $\overrightarrow{\mathbf{Y}}, \mathbf{Q}$, and $\mathbf{Q}^{\prime}$ respectively.

\section{AN INFORMATION Flow NumERICAL EXAMPLE}

The application of the FBIT method to the study of uncertainty propagation is now illustrated within a simple radar sensor example. An information loss budget is constructed for a baseline design. Selected forms of uncertainty in Table I are introduced into the system to demonstrate the analysis of the effects of propagating uncertainty through the information sensing channel.

\section{A. Radar Sensor and Target Model}

Using scattering center modeling and the far field approximation, the scattered field associated with the target can be modeled at the output of a radar step frequency measurement system for the specified target aspect angle $\left(\theta_{i}, \phi_{i}\right)$ [22]. The measured and signal processed random signature process $\overrightarrow{\mathbf{X}}_{\mathrm{n}}^{i}$ is then defined as in (21) where $\overrightarrow{\mathbf{n}}$ is additive white noise [12] modeled as the sum of thermal white noise and quantization noise components.

$$
\overrightarrow{\mathbf{X}}_{\mathrm{n}}^{\mathrm{i}}=\overrightarrow{\mathrm{X}}_{\mathrm{E}}^{\mathrm{i}}+\overrightarrow{\mathbf{n}}
$$

TABLE II. SENSOR SUMMARY

\begin{tabular}{|c|c|}
\hline Center frequency & $9.6 \mathrm{GHz}$ \\
\hline Transmit Bandwidth & $800 \mathrm{MHz}$ \\
\hline Number Bits in A/D Conversion & $8 \mathrm{Bits}$ \\
\hline Number of Pulses Integrated & 1024 \\
\hline $\begin{array}{c}\text { Signal-to-Noise Ratio } \\
\text { (time delay domain) }\end{array}$ & $\begin{array}{c}20 \mathrm{~dB} \\
\text { (variable) }\end{array}$ \\
\hline
\end{tabular}

1) Modeling Pose Angle Estimation Parameters in $\vec{V}_{t}$

The parameters $\sigma_{t}$ and $\mu_{t}$ are elements of $\vec{V}_{t}$ and are the standard deviation and bias of the sensor estimate of the object aspect angle respectively. The parameters $\theta$ and $\phi$ are both modeled $N\left(\sigma_{t}=.75^{\circ},\left\{\mu_{t}+\theta_{t}, \mu_{t}+\phi_{t}\right\}\right)$. The unknown bias parameter $\mu_{t}$ is modeled $U(-1,1)$ degrees.

\section{2) Modeling Leading Edge Estimation Parameters in $\vec{V}_{t}$}

The range alignment (along $\vec{l}$ os ) of the measured signature process $\overrightarrow{\mathbf{X}}$ to the decision rule training process $\overrightarrow{\mathbf{X}}^{\prime}$ is imperfect and can be modeled through a positive bias $\mu_{r}$ assumed to be distributed $U(0, .2)$ meters. The bias is applied to the phase center of the scattering cluster underlying $\overrightarrow{\mathbf{X}}$.

\section{3) Modeling Imperfect Training}

The dissimilarity between $\overrightarrow{\mathbf{X}}$ with $\overrightarrow{\mathbf{X}}^{\prime}$ will be generated using a matched scattering center model configuration with $\overrightarrow{\mathbf{X}}$. The uncertain parameters of $\vec{V}_{t}$ and $\vec{V}_{E}$ modeled within $\overrightarrow{\mathbf{X}}$ are not modeled in $\overrightarrow{\mathbf{X}}^{\prime} . \quad \overrightarrow{\mathbf{X}}^{\prime}=\overrightarrow{\mathbf{X}}$ only when $\overrightarrow{\mathbf{X}}$ is used directly for the training of the decision rule $d$.

\section{B. Feature Discriminate and Decision Rule Design}

The function $f$ used to compute the feature discriminate $\overrightarrow{\mathbf{Y}}$ in Fig. 1 is developed from the squared error of the distance from the mean templates $\vec{\mu}_{\overrightarrow{\mathbf{x}}_{1}}$ and $\vec{\mu}_{\overrightarrow{\mathbf{x}}_{2}}$ derived from the marginal training processes $\overrightarrow{\mathbf{X}}_{1}^{\prime}$ and $\overrightarrow{\mathbf{X}}_{2}$. The Maximum Likelihood estimator is used to determine the optimal decision rule $d$ assuming equally likely priors on each of the binary hypotheses $\mathrm{H}_{1}$ and $\mathrm{H}_{2}$ in $\overrightarrow{\mathbf{X}}$ and $\overrightarrow{\mathbf{Y}}$.

\section{EXPERIMENTS}

\section{A. Information and Design Trades in the Radar Channel}

The value of the Data Processing Inequality is readily seen from Fig. 2-4 below where the individual loss at each link in the channel can be quantified. In each of the figures, the MI and probability of error is computed for a changing design 
parameter within $v_{c}$, while uncertainty parameters within $\vec{V}_{t}$ and $\vec{V}_{E}$ are set to $\mu_{r}=\mu_{t}=0$ and $\overrightarrow{\mathbf{X}}=\overrightarrow{\mathbf{X}}^{\prime}$. Three design parameters are traded; system thermal noise, system dynamic range, and system bandwidth.

In Fig. 2 thermal noise is scaled by varying the noise figure across a range that affects a signal-to-noise-ratio (SNR in frequency domain prior to inverse Fourier Transform gain) range of $1 \mathrm{~dB}$ to $10 \mathrm{~dB}$. The results of the SNR trade indicate that increasing the SNR beyond $8 \mathrm{~dB}$ in the frequency domain (19 dB in the time-delay domain after transform gain) should further improve the information flow but only marginally.

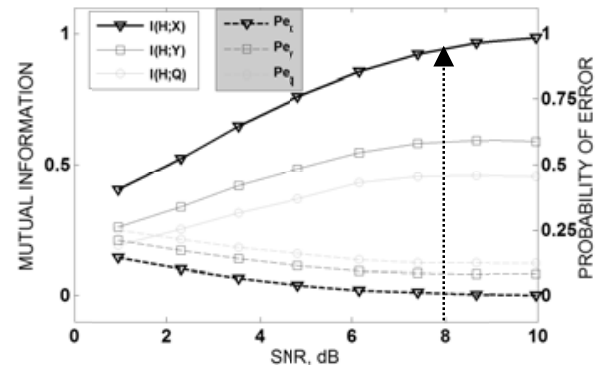

Fig.2. System Thermal Noise Trade, SNR in Frequency Domain

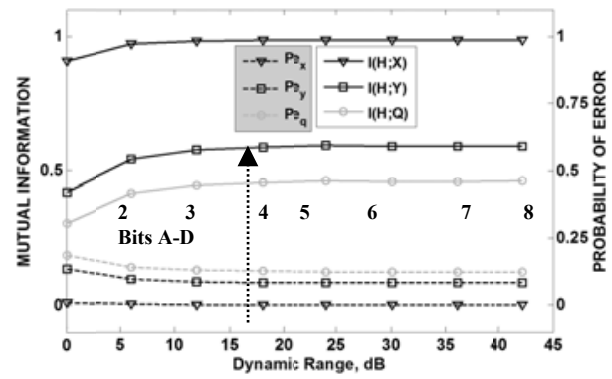

Fig. 3. System Dynamic Range Trade

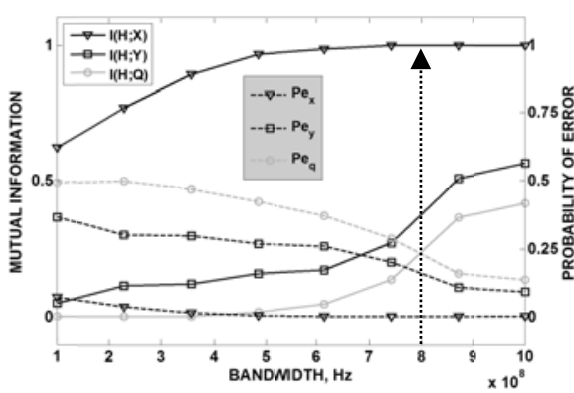

Fig. 4. System Bandwidth Trade

The sensitivity of $\mathrm{I}(\mathbf{H} ; \mathbf{Q})$ and ultimately $\mathrm{P}_{\mathrm{e}}$ to the dynamic range in the sensor is also of interest. The effects of quantization due to the twos-complement rounding error are assumed to be zero mean white noise processes [26]. The A/D quantization noise is modeled as an additive noise component [27],[28],[29] and is assumed to be uncorrelated with both the signal and the thermal noise. The trade in Fig. 3 indicates that a 4 Bit $\mathrm{A} / \mathrm{D}$ converter is needed to maximize information flow in the channel given the binary target set under evaluation.

The analysis of the bandwidth trade in Fig. 4 can be linked to the physical scattering configurations of target 1 and target 2. Locations for the non-collocated dominant scatterer differ by .2 meters or .65 feet. The theoretical resolution to resolve these two features would be approximately $800 \mathrm{MHz}$ using the fundamental bandwidth/resolution relationship. In Fig. 4 the bump in performance is centered at $800 \mathrm{MHz}$ where the mutual information at $\overrightarrow{\mathbf{Y}}$ and $\mathbf{Q}$ is rapidly increasing.

With one Bit going into the channel (binary classification problem), Table IV below tabulates the information loss budget for each trade study. Note the targets appear to be separating very well at $\overrightarrow{\mathbf{X}}$ while $.4-.5$ bits of loss have been sustained at the decision stage of the system, resulting in an "upper bound" in performance of $\mathrm{P}_{\mathrm{e}}=1$. No improvements to the classifier design can improve upon this performance level.

TABLE IV. INFORMATION LOSS BUDGET FOR VARIOUS TRADES

\begin{tabular}{|c|c|c|c|}
\hline \multirow{2}{*}{$\begin{array}{c}\text { System } \\
\text { Component }\end{array}$} & \multicolumn{3}{|c|}{ Information Loss, Bits } \\
\hline & $\begin{array}{c}\text { Trade } 1 \\
\text { SNR }\end{array}$ & $\begin{array}{c}\text { Trade } 2 \\
\text { DR }\end{array}$ & $\begin{array}{c}\text { Trade } 3 \\
\text { BW }\end{array}$ \\
\hline $\begin{array}{l}\text { Source-to- } \\
\text { Measurement }(\overrightarrow{\mathbf{X}})\end{array}$ & 0.1 & 0.1 & 0.05 \\
\hline $\begin{array}{l}\text { Measurement -to- } \\
\text { Feature }(\overrightarrow{\mathbf{Y}})\end{array}$ & 0.4 & 0.3 & 0.4 \\
\hline $\begin{array}{l}\text { Decision Rule } \\
\text { Application }(\mathbf{Q})\end{array}$ & 0.1 & 0.2 & 0.1 \\
\hline $\begin{array}{l}\text { Total Channel } \\
\text { Loss* }\end{array}$ & 0.6 & 0.6 & 0.55 \\
\hline
\end{tabular}

*Baseline Conditions; SNR $=20 \mathrm{~dB}, \mathrm{BW}=800 \mathrm{MHz}, \mathrm{DR}=20 \mathrm{~dB}$

An optimal design operating point may be; (i) A/D converter with $B=4$ Bits, (ii) Receiver design which achieves $20 \mathrm{~dB}$ SNR under tactically significant conditions, and (iii) Transmit waveform with BW $>800 \mathrm{MHz}$.

\section{B. Information Flow and System Uncertainty}

Equation (20) is demonstrated for links $\overrightarrow{\mathbf{X}}, \overrightarrow{\mathbf{Y}}$, and $\mathbf{Q}$. Three independent sources of uncertainty are introduced in the system under optimal training conditions. The parameters $\sigma_{t}$, $\mu_{t}$ and $\mu_{r}$ are as defined previously in IV.1 and IV.2. The target range is also unknown introducing a third uncertainty in the SNR of the measured signature. Using Monte-Carlo simulation $L=100$ independent draws of an $N_{M}$ sample ensemble from $\overrightarrow{\mathbf{X}}$ are generated. The FBIT method is applied at each draw to generate $\mathbf{I} \mathbf{L}_{\overrightarrow{\mathbf{x}}}, \mathbf{I} \mathbf{L}_{\overrightarrow{\mathbf{Y}}}$, and $\mathbf{I} \mathbf{L}_{\mathbf{Q}}$. To illustrate (11) the individual contributions to the cumulative link loss variance are plotted in an incremental fashion in Fig. 5.

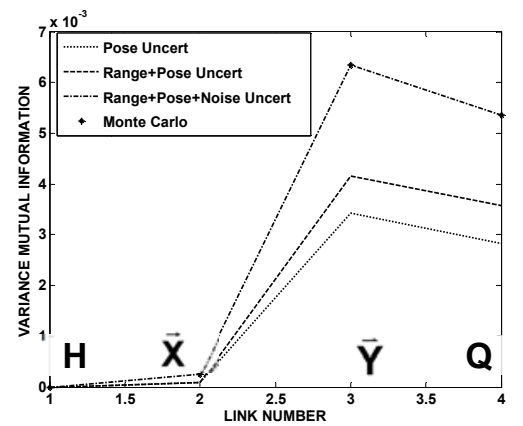

Fig. 5. Cumulative Channel Link Loss Variance, $L=100, N_{M}=3 * 10^{5}$

In Fig. 6 a similar example of the propagation of independent uncertainty sources is given for the variability in predicted performance demonstrating the use of (19). In Fig. 7 the cumulative link loss variance resulting from the sum of 
the independent three uncertainty sources is plotted about the expected cumulative link information. Applying (20) the combined impacts to the reliability in predicted link are presented in the error bars about the expected link performance. The implications of imperfect training are realized in the final stage of the channel at $\mathbf{Q}$ '.

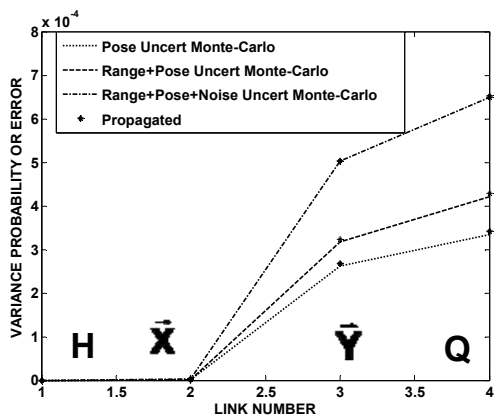

Fig. 6. Variability in Predicted Link Performance, $L=100, N_{M}=3 * 105$

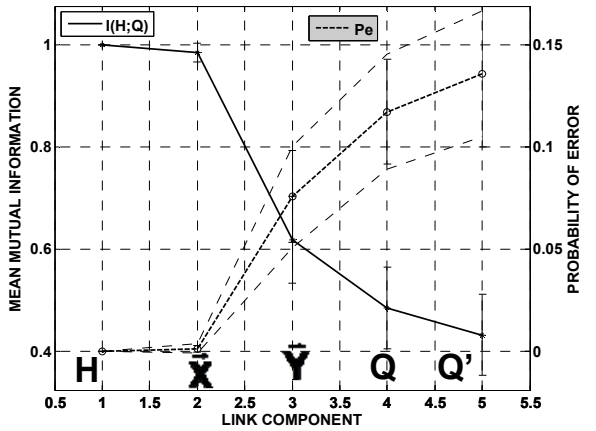

Fig. 7. Channel Mutual Information and Reliability in Link Performance, $\mathrm{L}=100, \mathrm{~N}_{\mathrm{M}}=3 * 10^{5}$

Table $\mathrm{V}$ below summarizes the link reliability and loss budget for the radar example. Note that $\mu_{\mathrm{IL}_{\text {Total }}}=\mu_{\mathrm{IL}_{\mathrm{Q}^{\prime}}}$.

TABLE V

INFORMATION RELIABILITY \& LOSS BUDGET FOR VARIOUS

\begin{tabular}{|c|c|c|c|}
\hline $\mathrm{L}$ & \multicolumn{3}{|c|}{ Link Information Measure } \\
\hline $\begin{array}{l}\mathrm{i} \\
\mathrm{n} \\
\mathrm{k}\end{array}$ & $\begin{array}{c}\text { Link Loss, } \\
\text { Bits }\end{array}$ & $\begin{array}{c}\text { Expected } \\
\text { Link } \\
\text { Performance }\end{array}$ & $\begin{array}{l}\text { Reliability in } \\
\text { Link } \\
\text { Performance }\end{array}$ \\
\hline $\bar{H}$ & 0.0 & -------- & ---- \\
\hline $\overrightarrow{\mathbf{x}}$ & $\mu_{\mathrm{IL}_{\mathrm{S}_{\Delta}}}=.05$ & $\mu_{\mathrm{P}_{\mathrm{e}_{\overline{\mathbf{x}}}}}=.013$ & $\sigma_{\mathrm{P}_{\overrightarrow{\mathrm{e}}}^{\overline{\mathrm{x}}}}=.003$ \\
\hline $\overrightarrow{\mathbf{Y}}$ & $\mu_{\mathrm{IL}_{\mathrm{F}}}=.35$ & $\mu_{\mathrm{P}_{\mathrm{e}_{\overline{\mathbf{Y}}}}}=.073$ & $\sigma_{\mathrm{P}_{\mathrm{e}}^{\overline{\mathrm{Y}}}}=.0228$ \\
\hline $\mathbf{Q}$ & $\mu_{\mathrm{IL}_{\mathrm{D} \Delta}}=.16$ & $\mu_{\mathbf{P}_{\mathbf{e}_{\mathbf{Q}}}}=.12$ & $\sigma_{\mathrm{P}_{\mathrm{e}}^{\mathrm{Q}}}=.0255$ \\
\hline $\mathbf{Q}^{\prime}$ & $\mu_{\mathrm{IL}_{\mathrm{T}_{\Delta}}}=.04$ & $\mu_{\mathrm{P}_{\mathrm{e}^{\mathrm{Q}^{\prime}}}}=.125$ & $\sigma_{\mathrm{P}_{\mathrm{e}}^{\mathrm{Q}^{\prime}}}=.0266$ \\
\hline
\end{tabular}

\section{CONCLUSION}

The FBIT method is developed for use in the research of information sensing applications. Measures are developed to identify information flow bottlenecks and to form an information link budget for system analysis. The FBIT method is applied to an HRR radar numerical example. The propagating effects of various sensing uncertainties on system performance are characterized at the component level.

\section{REFERENCES}

[1] P.M. Woodward, I.L Davies, "A Theory of Radar Information", Philosophical Magazine, Vol. 41, pp. 1001-1017, October 1951.

[2] P.M. Woodward, Probability \& Information Theory with Applications to Radar, 2nd edition, Pergamon Press, 1964

[3] C. E. Shannon, 'A mathematical theory of communication,' Bell System Technical Journal, Vol. 27, 379-423 and 623-656, July and Oct., 1948.

[4] M. Bell, "Information Theory and Radar: Mutual Information and the Design and Analysis of Radar Waveforms and Systems", Ph.D. Dissertation, Department of Electrical Engineering, California Institute of Technology, 1988.

[5] A. Leshem, O. Naparstek, A. Nehorai, "Information Theoretic Adaptive Radar Waveform Design for Multiple Extended Targets", IEEE Transactions on Selected Topics in Signal Processing, Vol. 1, 2007.

[6] S. Sowelam, A. Tewfik, "Waveform Selection in Radar Target Classification", IEEE Transactions on Information Theory, May 2000.

[7] S. Kullback, R.A. Leibler, "On Information \& Sufficiency", Ann. Math. Stat., 22: 79-86, 1951.

[8] S. Briles, "The Theory for, and the Demonstration of, Information Theory Applied to Radar Target Identification", Ph.D. Dissertation Kansas State University, 1992.

[9] M Horne "An Information Theory for the Prediction of SAR Target Classification Performance" DERA, Malvern, WR14 3PS, UK2, SPIE, Vol. 4382, 2001, Algorithms for Synthetic Aperture Radar Imagery VIII.

[10] J.C. Principe, D. Xu, Q. Zhao, J.W. Fisher "Learning form Examples with Information Theoretic Criteria", Journal of VLSI Signal Processing 26, 61-77, 2000.

[11] J. O'Sullivan, M. DeVove, V. Kedia, M. Miller, "SAR ATR Performance Using a Conditionally Gaussian Model", IEEE Transactions on Aerospace Electronics Systems Vol.37, No.1, Jan. 2001.

[12] K. Pasala, J. Malas, "HRR Radar Signature Database Validation for ATR; An Information Theoretic Approach", IEEE Transactions on Aerospace and Electronic Systems, Vol. 47, No. 2 April 2011.

[13] N. Tishby, F. Pereira, and W. Bialek. "The information bottleneck method" in Proc. 37th Allerton Conf. on Communication and Computation, 1999.

[14] C. H. Papadimitriou, K. Steiglitz, Combinatorial Optimization: Algorithms and Complexity "6.1 The Max-Flow, Min-Cut Theorem", pp. 120-128, Dover., 1998

[15] R. Ahlswede, N. Cai, S. Robert, and R. Yeung "Network Information Flow," IEEE Transactions on Information Theory Vol. 46, July. 2000.

[16] N. Merhav,"Data processing inequalities based on a certain structured class of information measures with application to estimation theory" IEEE Trans. Inform. Theory, vol. 58, no. 8, pp. 5287-5301, Aug. 2012.

[17] T. Santner, B. Williams, W. Notz, The Design and Analysis of Computer Experiments, Springer, 2003.

[18] B. Ayyub, G. Klir, Uncertainty Modeling and Analysis in Engineering and the Sciences, Chapman \& Hall/CRC, 2006.

[19] H. Coleman, W. Steele, Experimentation Validation, and Uncertainty Analysis, for Engineers, $3^{\text {rd }}$ Edition, John Wiley \& Sons 2009.

[20] J. A. Sokolowski, C. M. Banks, Modeling and Simulation Fundementals: Theoretical Underpinnnings and Pratical Domains, John Wiley \& Sons, 2010.

[21] A. Saltelli, et al, Global Sensitivitoes Analysis, The Primer, Wiley, 2008.

[22] J. Malas, J. Cortese, "The Radar Information Channel and System Uncertainty", IEEE Proceedings to the 2010 IEEE Radar Conference.

[23] T. Cover, J. Thomas, Elements of Information Theory, Wiley, 1991.

[24] J. A. Cortese, "Information Thoery and Radar Signal Processing", Air Force Research Lab Technical Report, Georgia Institute of Technology, College of Computing, March, 2009, Unpublished

[25] J. A. Cortese, MIT Lincoln Laboratory Technical Memorandums to AFRL/RYAS, 2011-2012, Unpublished.

[26] A. Oppenheim, R. Schafer, Discrete-Time Signal Processing, Second Edition, Prentice Hall, 1999.

[27] J. Scheer, J. Kurtz, Coherent Radar Performance Estimation, Artec House, 1993.

[28] M. Richards, Fundamentals of Radar Signal Processing, McGraw Hill, 2005.

[29] A. Oppenheim, Editor, Applications of Digital Signal Processing, Prentice Hall, 1978, page 663.

[30] Document approved for public release: $88 \mathrm{ABW}-2014-5992$, Dec 18, 2014 